need a considerable expansion of studies of African HIV isolates. In a review of variation in African HIV strains, Martine Peeters, Piot and Guido van der Groen discuss the need for further studies of the genetic and antigenic variation of HIV in different geographical regions. Vaccines will have to be effective against an array of isolates and it will be necessary to define the importance of genetic differences in relation to functional immune responses. West Africa also poses the problem of the HIV-2 virus; although there is increasing evidence that this is less pathogenic and less readily transmitted than HIV-1, the terminal stages of the infection are similar to those seen with HIV-1.

The review articles in the supplement to $A I D S$ highlight many other issues for the research agenda. But although agreeing that medical research is a priority, Barnett and Blaikie emphasize that this must be supplemented by studies of the economic and social impact of the

\section{Unpredictable reactions}

\author{
John J. Tyson
}

Chemical Chaos. By Stephen K. Scott. Oxford University Press: 1991. Pp. 454. £60, \$135.

CHAOS is a common part of everyday life. My desktop, toolbox and sock drawers are typically in a chaotic state. This type of chaos goes by the technical name 'entropy', which is very law-abiding, predictable stuff. But there is another type of randomness ('deterministic chaos') whose defining feature is not so much disarray as unpredictability. Deterministic chaos refers to a recurrent process that never exactly repeats itself. Furthermore, even though the process is governed by precise mathematical rules (such as some differential equations), its long-term evolution is irreproducible: two initial states, no matter how similar, will lead eventually to uncorrelated outcomes. Therefore, because the initial state of a real system can never be known with infinite precision, the longterm behaviour of a real chaotic system is fundamentally unpredictable.

That deterministic systems can behave unpredictably and irreproducibly is a blow to the scientific ethos to say the least. These revolutionary ideas first surfaced clearly in a toy mathematical model of fluid flow, in a theoretical study of biological population densities, and in experimental observations of some obscure chemical reactions.

Chemical chaos manifests itself as an epidemic and of the way in which affected African communities cope with its effects.

Writers in both volumes recognize that continued international support for control programmes and research on HIV in Africa is threatened; among the factors involved are an increasing complacency about the effects of the infection, a growing lack of interest in the problems of development in Africa, competition from other programmes and the absence of obvious, spectacular successes in AIDS control. Although the perspective and aims of these two volumes differ, readers of either will be convinced of the magnitude of the present and probable future effects of HIV infection in the continent and of the need for the wider international community to be more supportive of Africa's response to the pandemic.

H. A. Wilkins is in the Medical Research Council Laboratories, Fajara, PO Box 273, Banjul, The Gambia, West Africa.

irregular oscillation in some indicator of the progress of a chemical reaction, for instance a fluctuating electrode potential. The shining example of chemical chaos is the venerable BelousovZhabotinskii reaction (the oxidation of malonic acid by bromate, catalysed by iron ions) operating in a continuously stirred flow reactor at certain precise values of the flow rate. Irregular oscillations are also observed in gas-phase reactions (the oscillatory combustion of hydrogen and oxygen mixtures), in heterogenous processes (the oxidation of carbon monoxide on platinum crystals or the dissolution of copper electrodes) and in biochemical systems (the horseradish peroxidase reaction).

But what exactly is an 'irregular oscillation'? Is it a perfectly periodic oscillation that is randomly perturbed by the stirring and pumping machinery of the reactor, or could it be an almostperiodic oscillation similarly perturbed, or perhaps a complex concatenation of oscillatory states that would be perfectly periodic if only our experiments were accurate enough? Interesting as they may be, none of these possibilities is deterministic chaos as strictly defined. The fundamental challenge to proponents of chemical chaos is to prove that the irregular oscillations they observe are reflections of deterministic chaos in the underlying chemical reaction rather than random noise superimposed on a sensitive, but otherwise regular, chemical oscillator. This problem has bedevilled the field from the beginning and is still not totally resolved.

Scott's book is a serious and comprehensive introduction to chemical chaos. In language familiar to chemists, he explains all the essential theoretical ideas and important experimental examples. He wisely avoids technical details; readers who need to know how to calculate fractal dimensions or Lyapounov exponents can consult the literature cited. The book is a sequel to Chemical Oscillations and Instabilities, which he wrote with Peter Gray (Oxford University Press, 1990). Although the new volume can be read profitably on its own, the earlier volume presents a thorough overview of the exotic behaviour of nonlinear chemical reactions operating far from equilibrium: limit-cycle oscillations, multiple steady states and travelling waves, in addition to chaos. Serious students will want to own and study both volumes.

In Chemical Chaos, Scott reviews a broad selection of research articles and provides thorough reference lists. These services are useful to beginners trying to grasp the burgeoning literature on chemical chaos. Unfortunately, he does not give enough attention to some

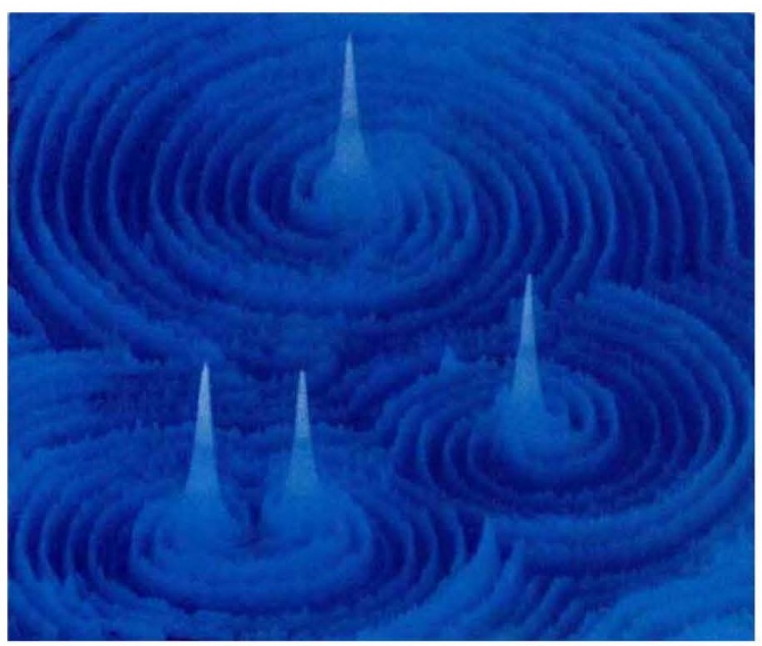

THE Belousov-Zhabotinskii reaction. The catalyst is usually a mixture of cerium (II) and (III) ions and ferroin, which acts as a visual indicator. The plate shows a typical reaction oscillating in composition and thus colour. The reaction is discussed in a chapter by Stephen Scott in The New Scientist Guide to Chaos edited by Nina Hall. Other contributors include lan Stewart, Robert May and Benoit Mandelbrot. Published by Penguin, $£ 9.99$ (pbk). 